\title{
Phonon-Assisted Two-Photon Interference from Remote Quantum Emitters
}

Marcus Reindl, $*^{\dagger} \dagger$ Klaus D. Jöns, ${ }^{*},{ }^{\ddagger}$ Daniel Huber, ${ }^{\dagger}$ Christian Schimpf, $^{\dagger}$ Yongheng Huo, $^{\S, \dagger, \|}$ Val Zwiller, ${ }^{\ddagger}$ Armando Rastelli, ${ }^{\dagger, \perp}$ and Rinaldo Trotta ${ }^{*}{ }^{\dagger}$

${ }^{\dagger}$ Institute of Semiconductor and Solid State Physics, Johannes Kepler University, Linz 4040, Austria

${ }^{\ddagger}$ Department of Applied Physics, Royal Institute of Technology, Stockholm 106 91, Sweden

${ }^{\S}$ Institute for Integrative Nanosciences, IFW, Dresden 01069, Germany

"Hefei National Laboratory for Physical Sciences at Microscale, University of Science and Technology, Shanghai 201315, China

${ }^{\perp}$ Johannes Kepler University, Linz Institute of Technology, Linz 4040, Austria

Supporting Information

ABSTRACT: Photonic quantum technologies are on the verge of finding applications in everyday life with quantum cryptography and quantum simulators on the horizon. Extensive research has been carried out to identify suitable quantum emitters and single epitaxial quantum dots have emerged as near-optimal sources of bright, ondemand, highly indistinguishable single photons and entangled photon-pairs. In order to build up quantum networks, it is essential to interface remote quantum emitters. However, this is still an outstanding challenge, as the quantum states of dissimilar "artificial atoms" have to be prepared on-demand with high fidelity and the
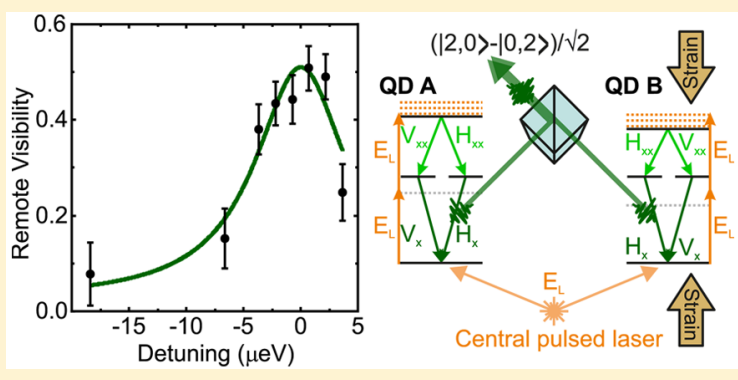
generated photons have to be made indistinguishable in all possible degrees of freedom. Here, we overcome this major obstacle and show an unprecedented two-photon interference (visibility of 51 $\pm 5 \%$ ) from remote strain-tunable GaAs quantum dots emitting on-demand photon-pairs. We achieve this result by exploiting for the first time the full potential of a novel phonon-assisted two-photon excitation scheme, which allows for the generation of highly indistinguishable (visibility of $71 \pm 9 \%$ ) entangled photon-pairs (fidelity of $90 \pm 2 \%$ ), enables push-button biexciton state preparation (fidelity of $80 \pm 2 \%$ ) and outperforms conventional resonant two-photon excitation schemes in terms of robustness against environmental decoherence. Our results mark an important milestone for the practical realization of quantum repeaters and complex multiphoton entanglement experiments involving dissimilar artificial atoms.

KEYWORDS: Quantum dots, quantum optics, two-photon interference, entanglement, resonant two-photon excitation

$\mathrm{O}$ ne of the very first requirements to observe ideal ondemand single photon emission ${ }^{1}$ is the population inversion of a two-level quantum system. Such a preparation of the quantum state is usually achieved via coherent excitation using resonant laser pulses, leading to an inverted two-level system performing Rabi oscillations. ${ }^{2}$ While single-pulse resonant excitation of a quantum dot (QD) has been used to achieve high state preparation fidelities and remarkable single photon properties, this scheme cannot be used to efficiently prepare the biexciton (XX) state, the key step to achieve polarization-entangled photon-pairs generation with QDs. This task can be instead accomplished using two-photon excitation $(\mathrm{TPE})^{3-5}$ techniques that have recently led to the generation of on-demand entangled photon pairs. ${ }^{6,7}$ This coherent excitation scheme, however, has one important drawback. Small fluctuations in the laser pulse area or energy as well as fluctuations in the $\mathrm{QD}$ environment result in a strong variation of the excited state population probability that, in turn, affects the efficiency of photon generation. Envisioned quantum communication and quantum simulation ${ }^{8}$ applications demand instead for more robust excitation schemes, being immune against these sources of "environmental decoherence" and ensuring on-demand generation of single and entangled photon-pairs. In principle, it is possible to overcome these problems by taking advantage of the solid state nature of QDs and in particular of their coupling to acoustic phonons. Although the phonon-assisted excitation scheme is inherently incoherent, it has been proposed ${ }^{9}$ and recently demonstrated ${ }^{10-12}$ that population inversion of exciton (X) and XX states coupled to a quasicontinuum of vibrational modes is indeed possible. Nonetheless, the capability of this technique to generate highly indistinguishable single and entangled photons has not been explored so far. In this Letter, we show for the first time that phonon-assisted two-photon excitation of QDs allows for the generation of highly indistinguishable entangled photonpairs. In comparison with standard excitation schemes, we

Received: February 22, 2017

Revised: $\quad$ May 17, 2017

Published: May 30, 2017 
a

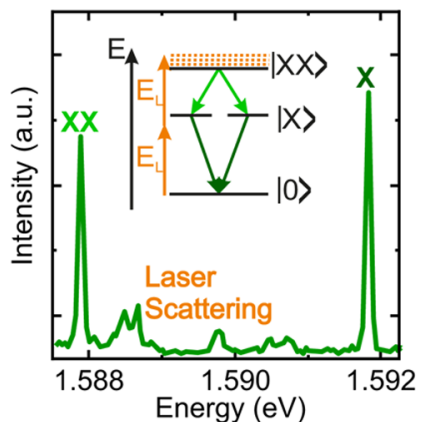

b

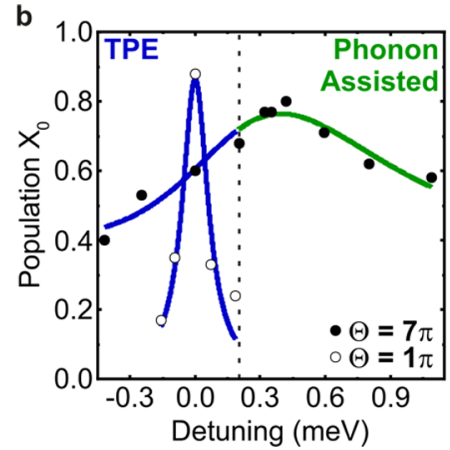

C

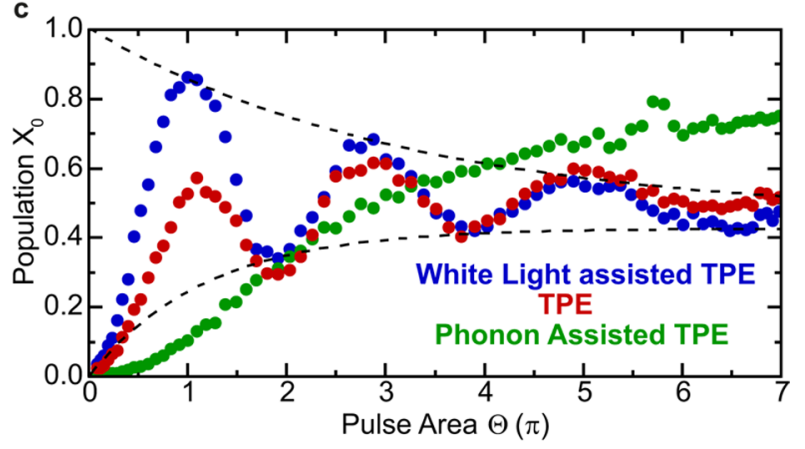

Figure 1. Spectrum and power dependent studies. (a) Spectrum of a GaAs QD under phonon-assisted TPE for optimized detuning of the laser energy and pulse length $\left(E_{\mathrm{L}}=1.5901 \mathrm{eV}, \tau_{\mathrm{p}}=10 \mathrm{ps}\right)$ and a pulse area of $6 \pi$. X and XX are clearly visible and the residual lines are attributed to laser scattering (mostly suppressed with notch filters without any polarization rejection) as well as weakly excited charged states (inset: sketch of the phonon-assisted two-photon excitation scheme used in this work. The dashed-orange lines represent the vibrational quasicontinuum coupled to the biexciton state). (b) Population of the X state as a function of the laser detuning for varying excitation power. While the traditional TPE (blue) suffers from a steep drop in inversion efficiency, a stable plateau can be observed exploiting the QD phonon sideband (green). The measured data for high excitation powers $(7 \pi)$ are interpolated with an asymmetric-modulated Gaussian function while the low power data $(\pi$-pulse) is fitted with a Lorentzian function. The two excitation regimes are distinguished considering the full width at half-maximum (fwhm) of the excitation laser $(\sim 0.2$ $\mathrm{meV}$ ). In particular, the dashed line indicates the configuration in which the detuning is set to a value that equals the fwhm. (c) Power dependent studies of the resonant TPE with (blue) and without (red) white light. The envelope of the Rabi oscillation is modeled with a single exponential damping. The results of the phonon-assisted excitation scheme are shown as green circles.

demonstrate that this method is more resilient against environmental decoherence limiting the $\mathrm{XX}$ or $\mathrm{X}$ preparation fidelity in conventional TPE schemes. Most importantly, we exploit its addressability with a wide-range of laser detunings to prepare on demand two remote and dissimilar QDs and to let the generated photons interfere at a beam splitter, a key experiment for the realization of an all-optical quantum repeater $^{13,14}$ and of photonic computing schemes. ${ }^{15}$

Results and Discussion. We focus our study on highly symmetric GaAs/AlGaAs QDs obtained via the droplet-etching method $^{16}$ (see Supporting Information (SI) Note 1). The photon-pairs emitted from this specific type of QDs have recently shown unprecedented high degree of entanglement as well as indistinguishability. ${ }^{7}$ A typical spectrum of our highly symmetric GaAs/AlGaAs QDs under phonon-assisted excitation is shown in Figure 1a. To address the vibrational modes coupled to the XX state, the excitation laser is blue detuned by $\Delta$ from the two-photon resonant case $(\Delta=0 \mathrm{meV})$ toward the $\mathrm{X}$ transition (Figure 1a). The best excitation parameters for optimum state preparation are inherently determined by the materials deformation potential and QD structural details, which determine the coupling of the exciton complexes to the acoustic phonons of the solid state environment, ${ }^{17}$ thus leading to the excitonic phonon sidebands. ${ }^{18}$ The optimal detuning energy for the investigated type of $\mathrm{QD}$ system is around $\Delta=$ $0.4 \mathrm{meV}$ for a pulse length of $\tau_{\mathrm{p}}=10 \mathrm{ps}$ (see SI Note 2). It is important to point out that the laser energy can be swept across a range of $0.2 \mathrm{meV}$ without perturbing the state preparation fidelity ( $5 \%$ population change, see SI Note 2 ), while in the conventional TPE the population varies by more than $80 \%$ on the same energetic range under $\pi$-pulse excitation (Figure $1 b$ ). The stability in the preparation fidelity versus the energy offered by the phonon-assisted scheme is particularly relevant for this work and will be later used to address remote QDs with the same laser. Before examining this point in more detail, we first discuss the robust nature of the phonon-assisted scheme in comparison with the standard TPE.

We first study the power dependence of the standard resonant TPE in the same excitation conditions and on the same QD (Figure 1c, red curve). Interestingly, the TPE manifests itself as oscillations of the state occupation probability pinned to $1 / 2$. While a possible explanation of this effect is the presence of a chirped laser pulse in conjunction with phononinduced damping, 9,19 we show that it is instead connected to the details of the QD environment. The power dependence changes considerably as we additionally illuminate the QD with a weak white-light source (Figure 1c, blue curve) revealing traditional phonon-damped Rabi oscillations ${ }^{20,21}$ with a maximum state population as high as $88 \pm 2 \%$. We attribute these modifications (which are particularly pronounced at the $\pi$-pulse) to saturation of crystal defects located in the vicinity of the QD. In the absence of the white light, these defects release/ trap charge carriers, thus giving rise to a fluctuating electric field. ${ }^{22}$ We hypothesize that the white light not only stabilizes the electric field experienced by the $\mathrm{QD}^{23}$ (see below) but also suppresses/saturates recombination channels (probably charged XX states) that hamper the radiative recombination of the XX into the $\mathrm{X}$ state. Obviously, for high values of the pulse area the effect becomes negligible as the carrier-phonon interaction dominates. While the use of an incoherent aboveband excitation is not generally required, many groups ${ }^{24-26}$ have reported that the ensuing charge stabilization dramatically improves the state preparation fidelities when the QDs are driven resonantly. Similarly, in the sample with GaAs/AlGaAs QDs employed here, we always find that the white light leads to an increase of the maximum population probability at the $\pi$ pulse with magnitudes that are QD dependent and that range from $10 \%$ to $50 \%$ (see Figure 1c). We also point out that the intensity of the white light needed to achieve the optimal conditions is also QD-specific. Thus, strict TPE schemes are not ideal for applications in complex networks and experiments with multiple sources. In stark contrast, no remarkable effect of the white light can be observed under phonon-assisted resonant excitation $(<5 \%$ change in preparation fidelity), probably due to the large laser power needed, which also stabilizes the environment. We would also like to emphasize that this is only one of the many advantages offered by the phononexcitation scheme. Beside its addressability to large laser 

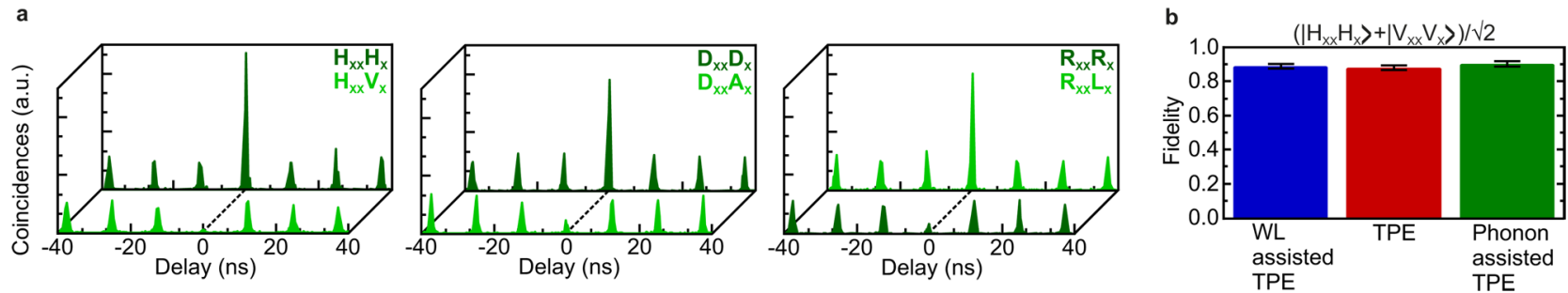

Figure 2. Comparison of entanglement. (a) XX-X cross-correlation measurements under phonon-assisted TPE for different polarization detection bases: rectilinear $(\mathrm{H}, \mathrm{V})$, diagonal $(\mathrm{D}, \mathrm{A})$ and circular basis $(\mathrm{R}, \mathrm{L})$. (b) Fidelity to the expected Bell state for all the different excitation methods.

detuning (see above), this scheme is inherently immune to fluctuations of the laser pulse area (see Figure 1c) due to its incoherent nature. More specifically, when the state preparation fidelity is maximum, a $10 \%$ fluctuation of the pulse area leads to a negligible $(<1 \%)$ change in the state population. In the standard TPE, the same fluctuation of pulse area gives rise to at least $7 \%$ variation in the state fidelity. Finally, we emphasize that the phonon-assisted scheme allows preparing the excited state with very high fidelity, which is as high as $80 \pm 2 \%$ for the highest laser pulse area available.

After demonstration of the robust nature of the phononassisted excitation scheme, we now investigate the quality of the generated photons in terms of entanglement fidelity and photon indistinguishability. We start out measuring the fidelity to the maximally entangled Bell state (see SI Notes 1 and 4) using a QD with small fine structure splitting (FSS) $(1.3 \pm 0.5$ $\mu \mathrm{eV})$. The polarization-resolved $\mathrm{XX}-\mathrm{X}$ cross-correlation measurements used to estimate the fidelity are shown in Figure 2a under phonon-assisted excitation. These data yield a fidelity of $f=90 \pm 2 \%$ which is identical (within the experimental error) to the values obtained when the $\mathrm{QD}$ is driven under strict TPE (with and without white light, Figure $2 \mathrm{~b}$ ). Therefore, the three different excitation schemes give rise to the same level of entanglement of the emitted photons. This is not surprising, as the fidelity is determined by three main contributions: (i) the relative value of the FSS with respect to the natural line width, ${ }^{27}$ (ii) recapture processes ${ }^{28,29}$ increasing the multiphoton emission and (iii) the hyperfine interaction. ${ }^{30,31}$ Because the lifetime (i) as well as the single photon purity (ii) and the hyperfine interaction (iii) are not affected by the excitation scheme (see SI Note 3), the fidelity to the Bell state is expected to remain constant, as indeed confirmed experimentally (Figure $2 b$ ).

The different excitation methods are instead expected to have a pronounced role in the indistinguishability of consecutive photons emitted by the same QD, as measured in a Hong$\mathrm{Ou}-$ Mandel type experiment ${ }^{32}$ on the XX and X photons. The time delay between consecutive photons was set to 2 ns via a Mach-Zehnder interferometer in the excitation path. The observation of the typical two-photon interference quintuplet is presented in Figure 3, together with the visibility values $V_{\mathrm{X}}$ and $V_{\mathrm{XX}}$, which are calculated taking into account the imperfections of the interference beam splitter and are referenced to the next neighboring side-peaks (see SI Notes 1 and 5). If we first take a look at the standard TPE (Figure 3a), we observe visibilities of $60 \%$ for both, XX and X photons, on the arbitrarily chosen QD. In particular, we would like to emphasize that the studied QD was selected randomly and that QDs with much higher visibilities can be found on the very same sample. ${ }^{7}$ The stabilization of the QD environment throughout illumination with the white light source (Figure $3 \mathrm{~b}$ ), however, leads to an a
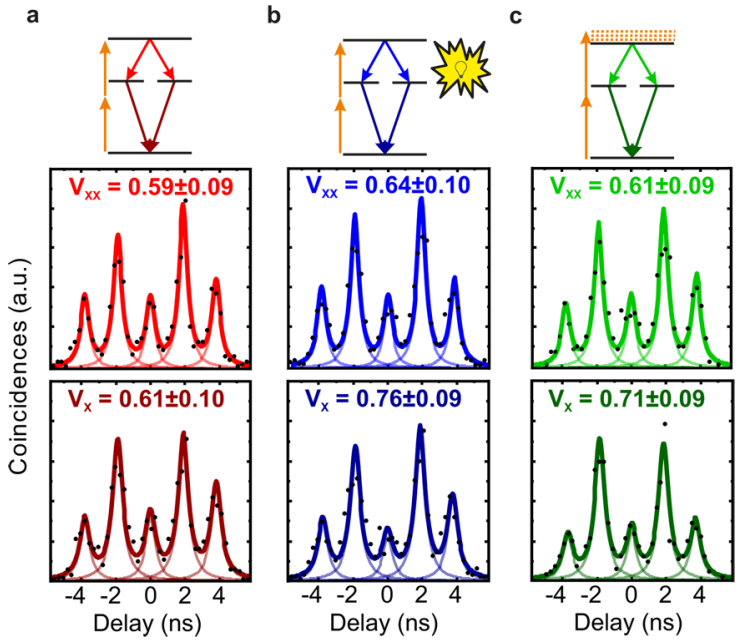

Figure 3. Two-photon interference using the same, randomly chosen, QD. The two-photon interference measurement in co-polarized configuration is performed on the same QD for (a) the standard TPE (b) the white-light-assisted TPE and (c) the phonon-assisted TPE, as schematically illustrated on top of each panel. The envelope function (bold) is the sum of 5 Lorentzian peaks fitted to the HongOu-Mandel quintuplet. The resulting interference visibilities are reported in each panel.

evident (slight) increase of the $\mathrm{X}(\mathrm{XX})$ visibility. This is reasonable as the $\mathrm{X}$ is more sensitive to spectral diffusion mediated by temporally charged defects ${ }^{22}$ than the screened potential of the fully occupied XX state. The weaker visibility of the XX transition, on the other hand, can be related to the XX probing an extraordinary noise environment ${ }^{33}$ and/or suffers from an initially higher pure dephasing rate. ${ }^{34}$ Most importantly, under phonon-assisted excitation of the two-level system (Figure 3c) a remarkably high level of indistinguishability (comparable to the TPE under white light illumination) can be observed. This demonstrates that the time jitter introduced by phonon relaxation has a negligible impact on our measured values of photon indistinguishabilities. This result is particularly relevant for applications, as the lifetime of the $\mathrm{X}$ and XX transitions in our GaAs QDs are very similar to the values reported for near-optimal single photon sources exploiting the Purcell effect and based on InAs QDs. ${ }^{35,36}$ Therefore, the fact that we do not observe a degradation in the photon indistinguishability suggests that the phonon-assisted excitation scheme may be readily adopted in systems showing the best performances in terms of indistinghuishability and brightness. ${ }^{37}$ To summarize, the phonon-assisted two-photon excitation scheme not only leads to the generation of highly indistinguishable entangled photon-pairs but it is more robust than the standard two-photon excitation schemes. Yet, this 
a

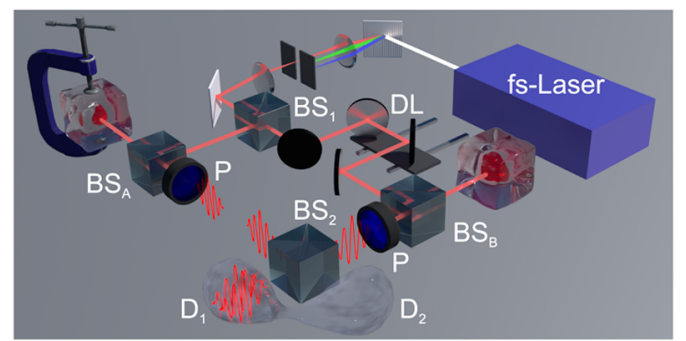

C

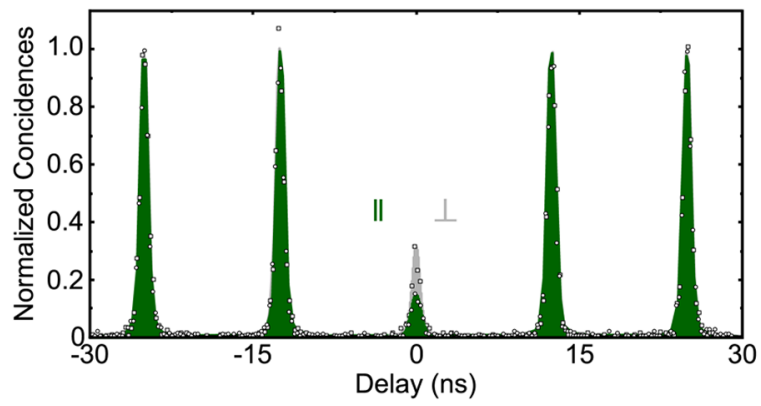

b

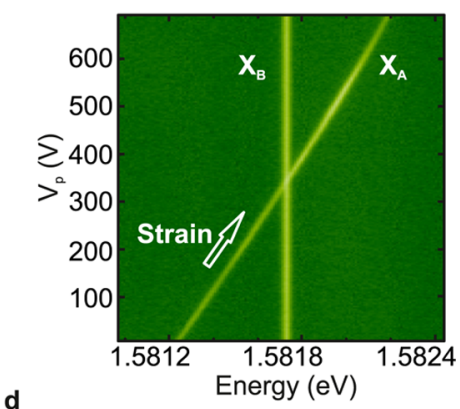

d

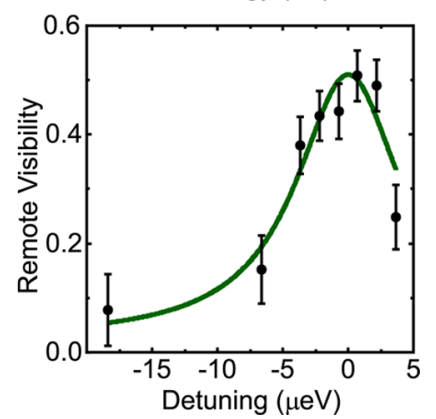

Figure 4. Two-photon interference from remote QDs. (a) Illustration of the interference of single photons from remote GaAs QD sources (ice cubes). A pulse-shaped femtosecond laser is split $\left(\right.$ at $\left.B S_{1}\right)$ and excites both QDs throughout excitation beam splitters $\left(\mathrm{BS}_{\mathrm{A}}\right.$ and $\mathrm{BS}_{\mathrm{B}}$, respectively) via the phonon-assisted TPE. The stream of photons from one of the QDs is mechanically delayed by a excitation-located delay line (DL) to ensure perfect timing coincidence for photons meeting at the interference beam splitter $\mathrm{BS}_{2}$. Strain tuning (c-clamp) of one $\mathrm{QD}$ allows for the precise frequency matching of the emitted photons, which are either co- or cross-polarized (using the polarizers $P$ ). Single-photon counters $\left(D_{1}\right.$ and $\left.D_{2}\right)$ are used to assess the quality of the interference by performing cross-correlation measurements at the two outputs of $\mathrm{BS}_{2}$. (b) Color-coded photoluminescence spectra of the $\mathrm{X}$ emission of QD A and QD B as a function of the piezo voltage $\left(V_{\mathrm{p}}\right)$ applied to QD A to achieve color coincidence with the $\mathrm{X}$ transition of QD B. (c) Second-order correlation for remote $\mathrm{X}$ photons excited in the phonon-assisted TPE scheme in coand cross-polarized configuration. (d) Remote two-photon interference visibility as a function of detuning between the two $\mathrm{X}$ photons.

scheme has an additional elegant advantage: it allows performing two-photon interference between remote QDs driven by the same pulsed laser of fixed frequency. This is a direct consequence of the wide range of laser detunings that allows achieving the maximum population inversion and it is in contrast to the traditional two-photon excitation schemes, which instead require a precise control of the laser energy for each individual QD. Since our QDs feature similar (albeit not identical) XX binding energies, the phonon-assisted twophoton excitation is a universal clocked excitation for arbitrary large numbers of QDs, hence a scalable approach for quantum optics.

The excitation of the remote emitters is timed so that the individual single photons from the two QDs, depicted as ice cubes in Figure $4 \mathrm{a}$, overlap on a beam splitter $\left(\mathrm{BS}_{2}\right)$ performing two-photon interference. For this experiment, we placed a second sample on top of a piezoelectric actuator to provide strain-tunability of the QD emission lines ${ }^{38-40}$ (see Methods) and to ensure frequency matching of the photons impinging at $\mathrm{BS}_{2}$. Two $\mathrm{X}$ transitions with almost identical lifetimes and high single photon purity from two remote QDs (see SI Note 6) are tuned to the same frequency by applying a voltage across the piezoelectric actuator mounted below QD A (Figure 4b). We stress once more that the standard TPE cannot be used to drive efficiently the two QDs, as the XX binding energies differ by more than $0.1 \mathrm{meV}$ (see the discussion above). Under phononassisted TPE we can instead prepare the two QDs with the highest probability by simply finding the optimum laser detuning for both, which in this special case turns out to be $\Delta=0.36 \mathrm{meV}$ at a pulse area of $5 \pi$ on each setup (Figure $1 \mathrm{~b}$ ), a value that is limited only by the power available in our experiment. The resulting correlation measurements are depicted in Figure $4 \mathrm{c}$ for the cross- and copolarized configurations. In contrast to the two-photon interference of consecutive photons from a single source, where the crosspolarized configuration always yields a value comparable to the side-peaks (see SI Note 5), this condition is not necessarily realized when combining remote single photon sources. In particular, long time-scale blinking (which may be due to random QD charging and consequent suppression of resonant absorption) reduces the value of $\mathrm{g}_{\perp}^{(2)}(0)$ even when the average intensities of the two emitters are kept the same. ${ }^{41}$ Thus, it is crucial to first determine the cross-polarized correlation and to evaluate the real remote two-photon interference visibility $V_{\text {remote }}$ as follows

$$
V_{\text {remote }}=\frac{g_{\perp}^{(2)}(0)-g_{\|}^{(2)}(0)}{g_{\perp}^{(2)}(0)}
$$

The optimized value for the overlap of the individual photon energies is then found by sweeping the $\mathrm{X}$ transition of QD A in steps of voltages that modify the energy on the order of a fraction of the line width, as demonstrated in Figure 4d. By doing so we report on a remote interference visibility as high as $V_{\text {remote }}=51 \pm 5 \%$, the highest value ever observed for $\mathrm{QDs}^{38,42-44}$ without the need of any temporal/spectral selection. So far only coherently scattered ${ }^{45}$ or Raman photons ${ }^{46}$ from remote QDs achieved higher two-photon interference visibilities. However, these excitation schemes cannot be used to generate pairs of photons and do not allow 
for the on-demand state preparation, both important prerequisites for quantum relays based on entangled photons from QDs. ${ }^{47}$ The measured visibility is in good agreement with the theoretical limits obtained from Michelson interferometry. Using a reported model ${ }^{44}$ and the QD parameters estimated in the experiment (see SI Note 6) we calculate that the maximum visibility achievable in the experiment is $56 \%$, a value which is very close to the experimental one. Currently we are only limited by the non-Fourier-limited photon emission of our QDs, probably due to charge noise and phonon sidebands, ${ }^{48}$ as we do not apply any spectral filtering in our measurement. A possible way to overcome these problems is to use devices that enable the application of electric fields ${ }^{49}$ and/or photonic cavities to shorten the lifetime of the transition via the Purcell effect, so as to effectively filter out the phonon sidebands.

In conclusion, we performed two-photon interference between photons emitted by two remote QDs with a visibility of $51 \pm 5 \%$, by using the full power of the novel phononassisted two-photon excitation scheme. In a comprehensive study, we compare different resonant excitation schemes and show that the phonon-assisted state preparation is a robust method to generate on-demand single pairs of highly entangled and indistinguishable photons from semiconductor quantum dots. It is interesting to note that this is the first report on twophoton interference using remote GaAs QDs and that the first (unsuccessful) attempt to observe this phenomenon with solidstate systems was also based on GaAs QDs in nanoholes. ${ }^{50}$ The proper choice of the QD growth strategy, ${ }^{51}$ of the energytuning method ${ }^{38,52}$ and, most notably, of the excitation scheme paves the way toward entanglement swapping experiments between independent QDs and other complex multisource experiments.

\section{ASSOCIATED CONTENT}

\section{S Supporting Information}

The Supporting Information is available free of charge on the ACS Publications website at DOI: 10.1021/acs.nanolett.7b00777.

Methods, detuning parameters of the phonon-assisted TPE; lifetime and autocorrleation; fidelity evaluation; two-photon interference using the same QD; two-photon interference from remote QDs (PDF)

\section{AUTHOR INFORMATION}

\section{Corresponding Authors}

*(M.R.) E-mail: marcus.reindl@jku.at.

*(K.D.J.) E-mail: klausj@kth.se.

*(R.T.) E-mail: rinaldo.trotta@jku.at.

\section{ORCID}

Marcus Reindl: 0000-0001-7333-515X

Klaus D. Jöns: 0000-0002-5814-7510

\section{Author Contributions}

M.R., K.D.J., and D.H performed the measurements with the help from C.S. and R.T. M.R. made the data analysis with the help from K.D.J., D.H., and R.T. Y.H. grew the sample with advice of A.R. C.S. processed the sample. M.R., K.D.J., and R.T. wrote the manuscript with the help from all the authors. R.T. conceived the experiment and supervised the project.

\section{Notes}

The authors declare no competing financial interest.

\section{ACKNOWLEDGMENTS}

This work was financially supported by the European Research Council (ERC) under the European Union's Horizon 2020 research and innovation programme (SPQRel, Grant Agreement 679183) and European Union Seventh Framework Programme (FP7/2007-2013) under Grant Agreement 601126 (HANAS). K.D.J. acknowledges funding from the MARIE SKŁODOWSKA-CURIE Individual Fellowship under REA Grant Agreement 661416 (SiPhoN). K.D.J. and R.T. acknowledge the COST Action MP1403, supported by COST (European Cooperation in Science and Technology). A.R. acknowledges funding from the Austrian Science Fund (FWF): P 29603. We acknowledge Florian Sipek and Matthias Gartner for help with the experimental setup and data evaluation. We further thank Javier Martin-Sanchez and Johannes Wildmann for fruitful discussions as well as Oliver G. Schmidt for providing the excellent sample used in this work.

\section{REFERENCES}

(1) He, Y.-M.; He, Y.; Wei, Y.-J.; Wu, D.; Atatüre, M.; Schneider, C.; Höfling, S.; Kamp, M.; Lu, C.-Y.; Pan, J.-W. Nat. Nanotechnol. 2013, 8, 213-217.

(2) Stievater, T. H.; Li, X.; Steel, D. G.; Gammon, D.; Katzer, D. S.; Park, D.; Piermarocchi, C.; Sham, L. J. Phys. Rev. Lett. 2001, 87, 133603.

(3) Brunner, K.; Abstreiter, G.; Böhm, G.; Tränkle, G.; Weimann, G. Phys. Rev. Lett. 1994, 73, 1138-1141.

(4) Stufler, S.; Machnikowski, P.; Ester, P.; Bichler, M.; Axt, V. M.; Kuhn, T.; Zrenner, A. Phys. Rev. B: Condens. Matter Mater. Phys. 2006, $73,125304$.

(5) Jayakumar, H.; Predojevic, A.; Huber, T.; Kauten, T.; Solomon, G. S.; Weihs, G. Phys. Rev. Lett. 2013, 110, 135505.

(6) Müller, M.; Bounouar, S.; Jöns, K. D.; Glässl, M.; Michler, P. Nat. Photonics 2014, 8, 224-228.

(7) Huber, D.; Reindl, M.; Huo, Y.; Huang, H.; Wildmann, J. S.; Schmidt, O. G.; Rastelli, A.; Trotta, R. Nat. Commun. 2017, 8, 15506.

(8) Aspuru-Guzik, A.; Walther, P. Nat. Phys. 2012, 8, 285-291.

(9) Glässl, M.; Barth, A. M.; Axt, V. M. Phys. Rev. Lett. 2013, 110, 147401.

(10) Ardelt, P.-L.; Hanschke, L.; Fischer, K. A.; Müller, K.; Kleinkauf, A.; Koller, M.; Bechtold, A.; Simmet, T.; Wierzbowski, J.; Riedl, H.; et al. Phys. Rev. B: Condens. Matter Mater. Phys. 2014, 90, 241404.

(11) Quilter, J. H.; Brash, A. J.; Liu, F.; Glässl, M.; Barth, A. M.; Axt, V. M.; Ramsay, A. J.; Skolnick, M. S.; Fox, A. M. Phys. Rev. Lett. 2015, 114, 137401.

(12) Bounouar, S.; Müller, M.; Barth, A. M.; Glässl, M.; Axt, V. M.; Michler, P. Phys. Rev. B: Condens. Matter Mater. Phys. 2015, 91, 161302.

(13) Duan, L.-M.; Lukin, M. D.; Cirac, J. I.; Zoller, P. Nature 2001, 414, 413-418.

(14) Sangouard, N.; Simon, C.; de Riedmatten, H.; Gisin, N. Rev. Mod. Phys. 2011, 83, 33-80.

(15) Knill, E.; Laflamme, R.; Milburn, G. J. Nature 2001, 409, 46-52.

(16) Huo, Y. H.; Rastelli, A.; Schmidt, O. G. Appl. Phys. Lett. 2013, 102,152105 .

(17) Krummheuer, B.; Axt, V. M.; Kuhn, T. Phys. Rev. B: Condens. Matter Mater. Phys. 2002, 65, 195313.

(18) Besombes, L.; Kheng, K.; Marsal, L.; Mariette, H. Phys. Rev. B: Condens. Matter Mater. Phys. 2001, 63, 155307.

(19) Reiter, D. E.; Lüker, S.; Gawarecki, K.; Grodecka-Grad, A.; Machnikowski, P.; Axt, V. M.; Kuhn, T. Acta Phys. Pol., A 2012, 122, 1065-1068.

(20) Förstner, J.; Weber, C.; Danckwerts, J.; Knorr, A. Phys. Rev. Lett. 2003, 91, 127401.

(21) Ramsay, A. J.; Gopal, A. V.; Gauger, E. M.; Nazir, A.; Lovett, B. W.; Fox, A. M.; Skolnick, M. S. Phys. Rev. Lett. 2010, 104, 017402. 
(22) Kamada, H.; Kutsuwa, T. Phys. Rev. B: Condens. Matter Mater. Phys. 2008, 78, 155324 .

(23) Gazzano, O.; Michaelis de Vasconcellos, S.; Arnold, C.; Nowak, A.; Galopin, E.; Sagnes, I.; Lanco, L.; Lemaître, A.; Senellart, P. Nat. Commun. 2013, 4, 1425.

(24) Jahn, J.-P.; Munsch, M.; Béguin, L.; Kuhlmann, A. V.; Renggli, M.; Huo, Y.; Ding, F.; Trotta, R; Reindl, M.; Schmidt, O. G.; et al. Phys. Rev. B: Condens. Matter Mater. Phys. 2015, 92, 245439.

(25) Bennett, A. J.; Lee, J. P.; Ellis, D. J. P.; Meany, T.; Murray, E.; Floether, F. F.; Griffths, J. P.; Farrer, I.; Ritchie, D. A.; Shields, A. J. Science Advances 2016, 2, 1501256.

(26) Gazzano, O.; Huber, T.; Loo, V.; Polyakov, S.; Flagg, E. B.; Solomon, G. S. 2017, arXiv e-prints 1702.03947. (Accessed Feb 15, 2017).

(27) Stevenson, R. M.; Hudson, A. J.; Bennett, A. J.; Young, R. J.; Nicoll, C. A.; Ritchie, D. A.; Shields, A. J. Phys. Rev. Lett. 2008, 101, 170501.

(28) Dousse, A.; Suffczyński, J.; Beveratos, A.; Krebs, O.; Lemaître, A.; Sagnes, I.; Bloch, J.; Voisin, P.; Senellart, P. Nature 2010, 466, 217-220.

(29) Jöns, K. D.; Schweickert, L.; Versteegh, M. A. M.; Dalacu, D.; Poole, P. J.; Gulinatti, A.; Giudice, A.; Zwiller, V.; Reimer, M. E. Scientific Reports 2017, 7, 1700.

(30) Deng, C.; Hu, X. Phys. Rev. B: Condens. Matter Mater. Phys. 2006, 73, 241303.

(31) Chekhovich, E. A.; Makhonin, M. N.; Tartakovskii, A. I.; Yacoby, A.; Bluhm, H.; Nowack, K. C.; Vandersypen, L. M. K. Nat. Mater. 2013, 12, 494-504.

(32) Hong, C. K.; Ou, Z. Y.; Mandel, L. Phys. Rev. Lett. 1987, 59, 2044-2046.

(33) Kuhlmann, A. V.; Houel, J.; Ludwig, A.; Greuter, L.; Reuter, D.; Wieck, A. D.; Poggio, M.; Warburton, R. J. Nat. Phys. 2013, 9, 570575.

(34) Vagov, A.; Axt, V. M.; Kuhn, T. Phys. Rev. B: Condens. Matter Mater. Phys. 2003, 67, 115338.

(35) Somaschi, N.; Giesz, V.; Santis, L. D.; Loredo, J.; Almeida, M.; Hornecker, G.; Portalupi, S.; Grange, T.; Antón, C.; Demory, J.; et al. Nat. Photonics 2016, 10, 340-345.

(36) Ding, X.; He, Y.; Duan, Z.-C.; Gregersen, N.; Chen, M.-C.; Unsleber, S.; Maier, S.; Schneider, C.; Kamp, M.; Höfling, S.; et al. Phys. Rev. Lett. 2016, 116, 020401.

(37) Claudon, J.; Bleuse, J.; Malik, N. S.; Bazin, M.; Jaffrennou, P.; Gregersen, N.; Sauvan, C.; Lalanne, P.; Gérard, J.-M. Nat. Photonics 2010, 4, 174-177.

(38) Flagg, E. B.; Muller, A.; Polyakov, S. V.; Ling, A.; Migdall, A.; Solomon, G. S. Phys. Rev. Lett. 2010, 104, 137401.

(39) Kumar, S.; Trotta, R.; Zallo, E.; Plumhof, J. D.; Atkinson, P.; Rastelli, A.; Schmidt, O. G. Appl. Phys. Lett. 2011, 99, 161118.

(40) Trotta, R.; Atkinson, P.; Plumhof, J. D.; Zallo, E.; Rezaev, R. O.; Kumar, S.; Baunack, S.; Schröter, J. R.; Rastelli, A.; Schmidt, O. G. Adv. Mater. 2012, 24, 2668-2672.

(41) Jöns, K. D.; Stensson, K.; Reindl, M.; Swillo, M.; Huo, Y.; Zwiller, V.; Rastelli, A.; Trotta, R.; Björk, G. 2017, arXiv e-prints 1702.03278. (Accessed Feb 13, 2017).

(42) Patel, R. B.; Bennett, A. J.; Farrer, I.; Nicoll, C. A.; Ritchie, D. A.; Shields, A. J. Nat. Photonics 2010, 4, 632-635.

(43) Gold, P.; Thoma, A.; Maier, S.; Reitzenstein, S.; Schneider, C.; Höfling, S.; Kamp, M. Phys. Rev. B: Condens. Matter Mater. Phys. 2014, 89, 035313.

(44) Giesz, V.; Portalupi, S. L.; Grange, T.; Antón, C.; de Santis, L.; Demory, J.; Somaschi, N.; Sagnes, I.; Lemaitre, A.; Lanco, L.; et al. Phys. Rev. B: Condens. Matter Mater. Phys. 2015, 92, 161302.

(45) Gao, W.; Fallahi, P.; Togan, E.; Delteil, A.; Chin, Y.; MiguelSanchez, J.; Imamoglu, A. Nat. Commun. 2013, 4, 2744.

(46) He, Y.; He, Y.-M.; Wei, Y.-J.; Jiang, X.; Chen, M.-C.; Xiong, F.L.; Zhao, Y.; Schneider, C.; Kamp, M.; Höfling, S.; et al. Phys. Rev. Lett. 2013, 111, 237403.

(47) Trotta, R.; Martin-Sanchez, J.; Daruka, I.; Ortix, C.; Rastelli, A. Phys. Rev. Lett. 2015, 114, 150502.
(48) Iles-smith, J.; Mccutcheon, D. P. S.; Nazir, A.; Mørk, J. 2016, arXiv e-prints 1612.04173. (Accessed Dec 14, 2016).

(49) Kuhlmann, A. V.; Prechtel, J. H.; Houel, J.; Ludwig, A.; Reuter, D.; Wieck, A. D.; Warburton, R. J. Nat. Commun. 2015, 6, 8204.

(50) Benyoucef, M.; Wang, L.; Rastelli, A.; Schmidt, O. G. Appl. Phys. Lett. 2009, 95, 261908.

(51) Heyn, C.; Stemmann, A.; Köppen, T.; Strelow, C.; Kipp, T.; Grave, M.; Mendach, S.; Hansen, W.; Heyn, C.; Stemmann, A.; et al. Appl. Phys. Lett. 2009, 94, 183113.

(52) Trotta, R.; Wildmann, J. S.; Zallo, E.; Schmidt, O. G.; Rastelli, A. Nano Lett. 2014, 14, 3439-3444. 